\title{
A real-time software voice key and an application
}

\author{
ROBERT SHERAK \\ University of Chicago, Chicago, Illinois 60637
}

\begin{abstract}
This paper describes a real-time software voice key that can be used to "locate" speech onset and offset within a 4-msec "window" and to distinguish among several classes of speech sounds (e.g., sibilance, nonsibilance, aspiration, and silence). It is particularly appropriate for applications in which the course of a program controlling an experiment is dependent on what, when, and how a subject utters. Additionally, an application of this voice key is outlined with the intent of suggesting other methodological possibilities and innovations to phoneticians and psychologists considering research projects in which the precise measurement of speech onset latency or the duration of specific speech segments, including pauses, is desired.
\end{abstract}

Hardware and software voice keys have become useful and sometimes necessary tools for researchers interested in both the nature of the speech signal and speech as an experimental dependent variable. Hardware voice keys have been used for at least the past 40 years (Border, 1939), and with the increasing use of computers for research, software voice keys have been designed (Martz \& Welkowitz, 1977; Wright, Note 1). For the most part, however, the development of software voice keys has been an "underground" phenomenon, with little of the necessary information and discussion for evaluating the effectiveness or the applicability of this software surfacing into the behavioral science journals. This paper attempts to address this situation by describing a software voice key and an experimental procedure that was greatly facilitated by this technology.

\section{THE VOICE KEY}

\section{Environment}

The voice key is realized as a MACRO (PDP-11 assembly language) subroutine running on a PDP-11/34 minicomputer, under the RT-11 operating system, that is equipped with a $\mathrm{KW} 11-\mathrm{K}$ real-time programmable clock and an $\mathrm{AD} 11-\mathrm{K}$ analog-to-digital (A/D) converter. The subroutine requires 500 bytes of memory in a "stand-alone" environment, and it uses $100 \%$ of the CPU time. It does not need hardware or software floating-point arithmetic, and all variables are integers ( 2 bytes) or less. Because of the "sensitivity" of the voice key, considerable effort was spent in minimizing both acoustic and electrical noise within the laboratory.

A representation of the speech waveform (AA), zero crossings (ZC), and indicators for speech onset and

The author wishes to acknowledge the helpful suggestions and criticisms of Doris Aaronson, Norman Brown, Jody Kreiman, Misha Pavel, Dale Terbeek, and Ted Wright. Requests for reprints should be sent to Robert Sherak, Department of Psychology, New York University, 6 Washington Place, New York, New York 10003. offset may be displayed. If this option is chosen, then it is necessary to use an AAl1-K to perform digital-toanalog (D/A) conversion and to control a Tektronix 607 storage monitor operating in store mode. Other hardware may be substituted where appropriate, or the subroutine may be modified for a new environment. Figure 1 is an annotated representation of how the storage monitor display might appear.

\section{The Algorithm}

The main body of the subroutine executes in a loop until speech onset or offset is detected or a specified number of milliseconds has elapsed, and then it returns control to the calling program. ${ }^{1}$ Simultaneously, A/D conversion takes place and the window statistics (explained below) are accumulated. The subroutine continually checks to see if the time frame for each window has ended and, if it has, examines two window statistics. For each window, which is a user-defined time period generally set between 4 and $20 \mathrm{msec}$, if either

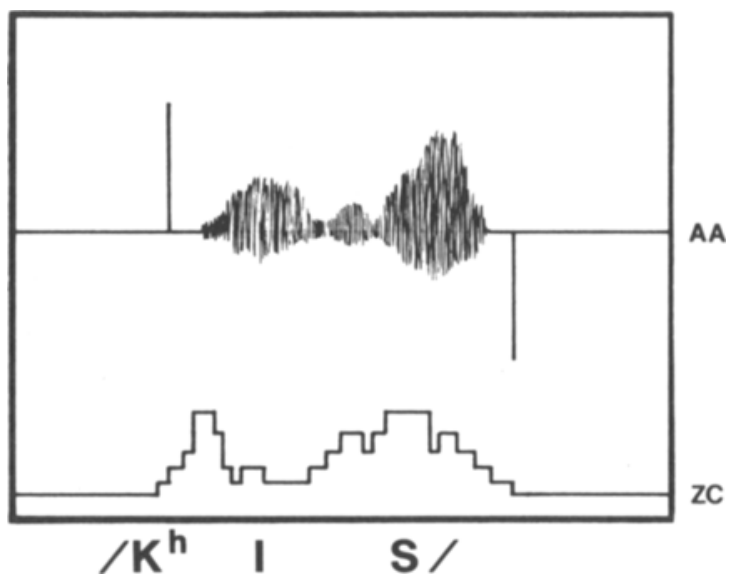

Figure 1. Annotated representation of the speech waveform (middle) and zero crossings per window (bottom) for the word "kiss." Two markers indicate speech onset and speech offset, respectively. In this example, the $\mathrm{ZC}$ threshold was exceeded before the AA threshold. 
statistic exceeds a threshold, then a "hit" is recorded. The presence or absence of speech is evaluated by determining if the count of consecutive hits exceeds a user-specified value. For detecting speech offset, the procedure is more complicated, as the subroutine executes for a specified number of milliseconds longer than the last detection of a run of hits (attempting to insure that the absence of a hit is not artifactual). The first statistic is based upon the absolute average amplitude (AA) and is useful for detecting relatively moderateand high-volume speech sounds, such as nasals and vowels. The second statistic is based upon the number of compression/rarefaction cycles ( $\mathrm{ZC})$ and is particularly useful for detecting relatively low-volume, aperiodic sounds such as voiceless fricatives and aspiration. Aspects of these procedures have been employed for various projects requiring signal detection, including research on EEG (Lubar \& Culver, 1978), EMG (Cohen, Note 2), speech production (Martz \& Welkowitz, 1977; Stemberg, Wright, Knoll, \& Monsell, 1980; Wright, Note 1), and speech recognition (Atal \& Rabiner, 1976).

\section{Asynchronous Sampling}

Every 100 microsec, the $\mathrm{KW} 11-\mathrm{K}$ clock initiates an A/D conversion (which occurs in parallel with the execution of the subroutine). Upon completion of the conversion (about 20 microsec later), an interrupt signal is issued by the $A / D$ converter. An interrupt service routine recodes the 12 bits of the converted signal into a number between $+2,047$ and $-2,047$ (a sample), which represents the amplitude of the signal. For an index of the AA component, the routine accumulates the scaled absolute value for each sample. For an index of the $\mathrm{ZC}$ activity, the routine compares the sign of each sample with that of its predecessor. If they differ, the $\mathrm{ZC}$ counter is incremented. This routine is also responible for updating the image on the storage monitor.

\section{Main Parameters}

The behavior of the subroutine depends on several parameter settings whose default values may be overridden by the user in the calling program. This section describes most useful parameters and their default values and indicates how these values were chosen for this implementation.

The parameter "window size" is defined in terms of the number of samples to be collected within it. Each sample increases the window size by $100 \mathrm{microsec}$ (as well as the amount of information about both the $\mathrm{AA}$ and $\mathrm{ZC}$ speech component). This parameter should be set large enough that the statistics for one window alone contain enough information to distinguish between a constant signal and silence, and small enough that it is appropriately precise for the voice key's application (the smaller the window, the greater the precision). It must also allow the subroutine (as opposed to the A/D interrupt service routine) sufficient time to perform its evaluative activity. The default for this param- eter, 40 samples, seems optimal, given these requirements, for most of my applications. It appears that while sampling at $10,000 \mathrm{~Hz} \quad 28$ samples/window approaches a minimum with respect to this last requirement (this estimate may be relevant to similar voice keys).

The parameters for the AA threshold (one for onset and another for offset) should be set proportional to the gain setting of the amplifier. Since the program variable tracking AA activity is only 16 bits, it may overflow if the window is too big or if the average amplitude of the signal is too high. Hence, there is a scaling parameter available as a divisor for each sample (with a default of one). Baseline values for the AA component without speech input run on the order of $80 \%$ less than the value recorded for the first window at the onset of an open vowel. The default for the parameters for the ZC threshold (one for onset and another for offset) is four. Baseline data indicate that $90 \%$ of the windows recorded without speech input have $\mathrm{ZC}$ values less than this default.

The number of consecutive hits defining speech onset and offset may be independently specified (both default values are six). The number of consecutive hits for onset must be large enough to minimize the probability of nonspeech sounds' being evaluated as speech. When the number of samples per window is 40 , six hits (which represents $24 \mathrm{msec}$ of speech) appears to be optimal for speech onset. For speech offset, this value may be best set lower; however, another parameter must be set concurrently. This parameter specifies the number of milliseconds after the detection of speech onset, and after the detection of a nonhit, that the subroutine should continue executing to be sure that speech has truly ceased. Its value should be set longer than the longest "natural" absence of sound (as in a stop consonant) but short enough to minimize the probability of having spurious noise or "posttrial" utterances detected as speech. Optimal settings for this parameter are on the order of $100 \mathrm{msec}$ for rapid speech and $500 \mathrm{msec}$ (the default) for "careful" speech.

Other parameters allow for flexibility in controlling the storage monitor, such as the number of milliseconds of speech to be displayed for each subroutine call, the display's location, and the scale. The A/D channel and the sample frequency may be set by the user. Additionally, the two statistics for each window may be saved in a buffer supplied by a calling program. This information is useful for calibrating the voice key to different operating environments.

\section{AN APPLICATION}

This subroutine facilitated the development of an experimental paradigm derived from similar procedures used by Ladefoged, Silverstein, and Papçun (1971, 1973) and Lindig, McNeill, and Stevens (Note 3), and described by Sherak (Note 4). Its purpose is to examine 


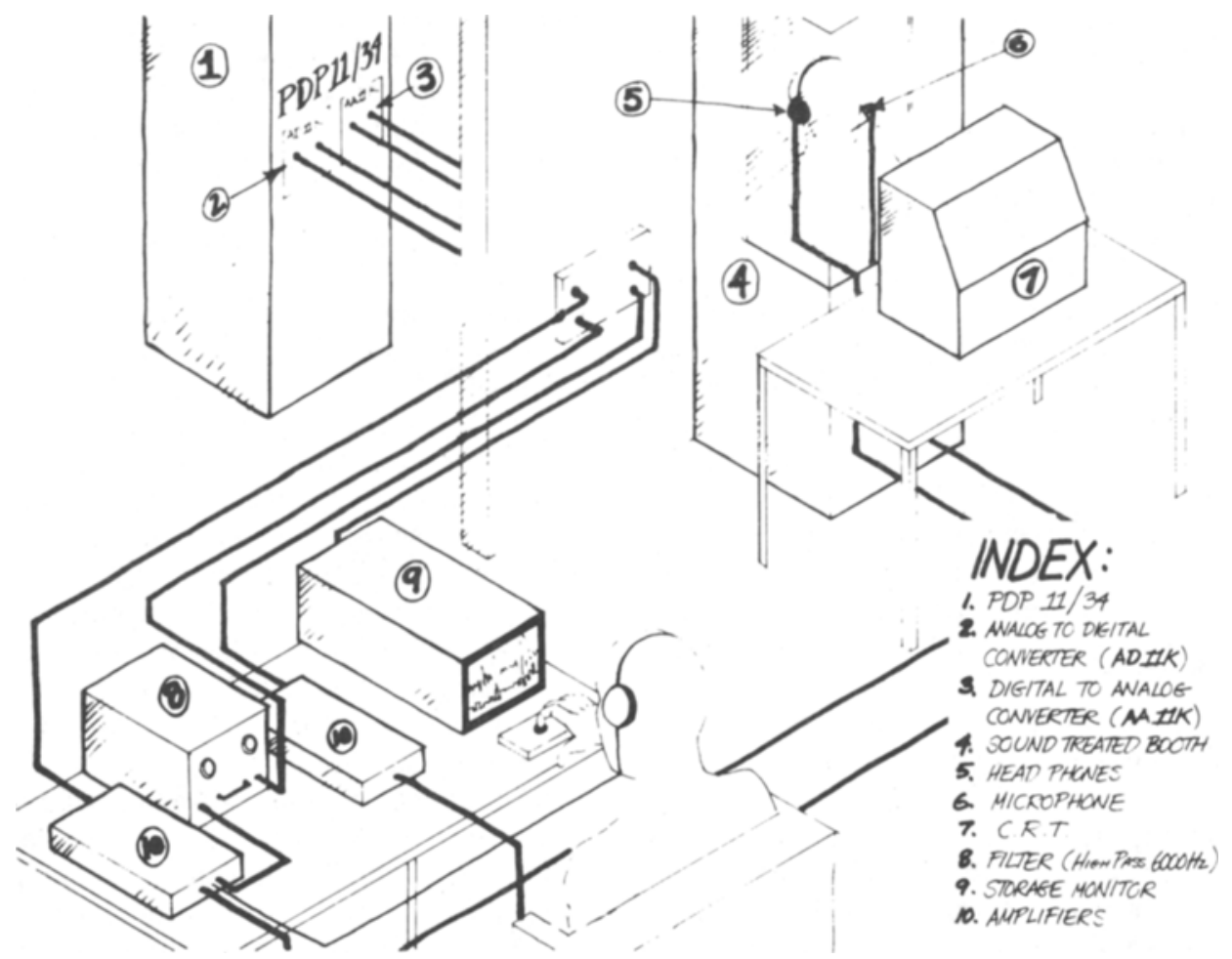

Figure 2. Typical laboratory arrangement for most experiments using this voice key.

speech production activity and the cognitive resources allocated to it in an on-line fashion. An index of this activity is compared for different sorts of linguistic material.

\section{Apparatus}

The arrangement of the experimental apparatus is schematically represented in Figure 2.

The subject sits in a sound-treated booth viewing a CRT that displays a phrase to be uttered; he or she wears a headset through which an auditory signal may be presented. Attached to the headset is a microphone into which the subject may speak. The microphone's output is split in two. One part goes directly into an A/D channel, and the other part is high-pass filtered at $6,000 \mathrm{~Hz}$ and then goes into an alternate $\mathrm{A} / \mathrm{D}$ channel. The experimenter sits at the table listening to the unfiltered speech, viewing the storage monitor, and, when appropriate, pressing a "feedback" button.

\section{Subject's Instructions}

Upon presentation of an auditory stimulus (start signal), subjects are instructed to immediately utter, at a "nomal pace," a well learned phrase that is displayed upon the CRT. Upon presentation of another auditory stimulus (stop signal), they are told to stop uttering the phrase and to utter/ps/(stop gesture) as soon as possible. Subjects are given extensive practice, and many trials are run for a variety of phrases. The course of a typical trial is schematically represented in Figure 3.

\section{The Main Program}

A FORTRAN program displays a phrase upon the subject's CRT. Then, after a delay of $500-800 \mathrm{msec}$, a $500-\mathrm{Hz}$ tone is presented to the subject for $25 \mathrm{msec}$ (start signal). The voice-key subroutine is called to determine the latency from the start signal to speech onset. When speech onset is detected, the subroutine returns control to the main program. For the "noncatch" trials, after a variable delay $(25-525 \mathrm{msec})$, a stop signal (a similar tone at 1,000 Hz) is presented to the subject. The subroutine again is called with a parameter set so that it will sample from the alternate $A / D$ channel. This channel contains the subject's speech high-pass filtered at $6,000 \mathrm{~Hz}$. (Although with the sampling rate at $10,000 \mathrm{~Hz}$ the subroutine is in effect subsampling from this channel, this does not seem to markedly affect the voice key's accuracy.) The subject's utterances contain little energy above $6,000 \mathrm{~Hz}$, but the stop gesture does, so only sound from this gesture passes through the filter to the A/D converter. Thus, the subroutine is able to detect the onset of sibilance in the stop gesture (from the alternate $\mathrm{A} / \mathrm{D}$ channel) and calculate

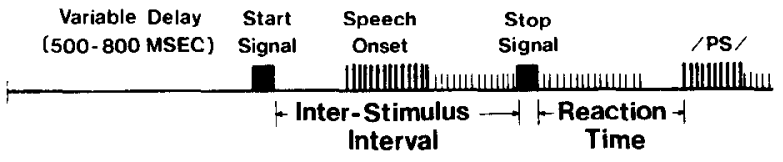

Figure 3. Representation of a typical trial. In most experiments, the reaction time to alter speech is examined in terms of interstimulus interval. 


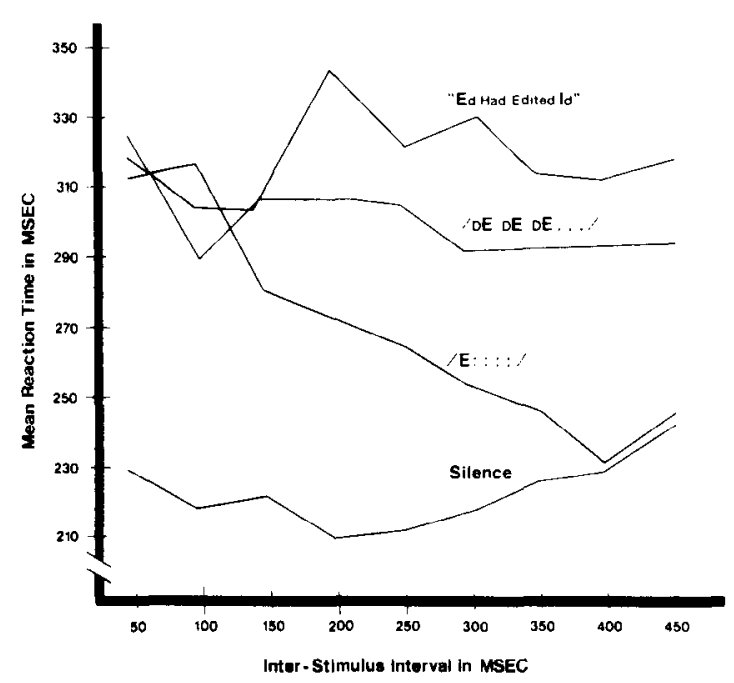

Figure 4. One result for eight subjects. Curves for mean RT as a function of interstimulus interval have a greater ordinate as "linguistic complexity" increases.

an index of the amount of time it takes the subject to switch from one utterance to another (switching time). The experimenter views the display to determine if the subject has performed accurately, as is the case over 95\% of the time. The program waits for a verification response from the experimenter's keyboard, which it records and uses to determine the course of the experiment.

In one analysis, the speech switching time is examined in terms of the interstimulus interval (the time between the start and stop signals) for four phrases that vary in "linguistic complexity." The result appears in Figure 4. The $\mathrm{x}$-axis represents the interstimulus interval and the $y$-axis represents switching time. The small yet significantly different levels and slopes among the curves illustrate the utility of a real-time voice key with this degree of precision and with the ability to distinguish certain speech sounds.

\section{CONCLUSION}

This application may suggest procedures appropriate to other experiments in which the course of a program controlling the experiment is dependent upon particular speech sounds uttered by the subject. For example, with the ability to classify an utterance by whether or not it contains high frequency sound, as in this example, which uses $/ \mathrm{ps} /$, a computer program controlling an experiment may distinguish between responses as "yes" or "no" and "true" or "false" and collect their latencies.

Further research in to speech physiology and "control of action," as well as into optimal voice-key algorithms, should lead to improvements in this technology and its appropriate applications. Electromyographic and $\mathrm{X}$-ray film studies, for example. may lead to a better understanding of the relationship between speech onset and offset as acoustically realized and the motor programming and motor activity that produced the speech.
The procedure for detecting low-amplitude speech sounds may also be improved. In certain circumstances, the ZC statistic beneficially can be replaced or augmented with other statistics, such as weighted (in a variety of ways) ZC or weighted or unweighted counts of local maxima (Atal \& Rabiner, 1976; Wright, Note 1). Additionally, the procedure for evaluating speech onset or offset can be based upon the standard deviation of statistics for a number of adjacent windows (Cohen, Note 2). Furthermore, with the increase in speed and power of computer hardware, more complicated algorithms may be employed, including procedures that easily allow for the subroutine to be called as an asynchronous process.

\section{REFERENCE NOTES}

1. Wright, C. Personal communication, November 1981.

2. Cohen, B. AVGEMG (PDP-12 computer program). New York University, Department of Psychology, Psychophysiology Laboratory, 1976.

3. Lindig, K., McNeill, D., \& Stephens, D. Induced interruptions of speech. Unpublished manuscript, University of Chicago, Committee on Cognition and Communication, 1980.

4. Sherak, R. Recent experiments on speech motor programming using reaction time techniques. Unpublished manuscript, New York University, Department of Psychology, 1981.

\section{REFERENCES}

Atal, B., \& Rabiner, L. A pattern recognition approach to voiced-unvoiced-silence classification with applications to speech recognition. IEEE Transactions on Acoustics, Speech and Signal Processing, 1976, ASSP-24, 201-212.

Bonder, D. A new apparatus for voice control of electric timers. Journal of Experimental Psychology, 1939, 26, 241-247.

Ladefoged, P., Silverste in, R., \& Papçun, G. Interruptibility of speech. U.C.L.A. Working Papers in Phonetics, 1971, 17, 85-94.

Ladefoged, P., Silverstein, R., \& Papçun, G. Interruptibility of speech. Journal of the Acoustic Society of America, 1973, 54, 1105-1108.

Lubar. J., \& Culver, R. Automated EEG signal-detection methodologies for biofeedback conditioning. Behavior Research Methods \& Instrumentation, 1978, 10, 607-616.

MarTz, M., \& WeLKowitz, J. WELMAR-Computer programs to analyze dialogic time patterns. Perceptual and Motor Skills, 1977, 45, 531-537.

Sternbera, S., Wright, C., Knoli, R., \& Monsell, S. Motor programs in rapid speech: Additional evidence. In $\mathbf{R}$. Cole (Ed.), Perception and production of fluent speech. Hillsdale, N.J: Erlbaum, 1980.

\section{NOTE}

1. My first exposure to voice-key technology was obtained while implementing, on a PDP-11/34, a version of this particular algorithm, which was originally developed and installed on a General Automation 18/30 computer by the Human InformationProcessing Research Department of Bell Laboratories, Murray Hill, New Jersey. The algorithm my subroutine employs is a modification that increases the number of parameters. This permits, for example, a smaller window size within which to locate speech. My version uses zero crossings instead of a measure of the magnitude of the derivative of the speech wave at its local maxima and includes certain display features and calibration statistics. I would like to acknowledge the important role of Saul Sternberg, Mel Melchner, and Charles E. Wright in developing this technology. A complete report of their work is in progress. 